\title{
Causal Compensation for Erasures in Frame Representations
}

\author{
Petros Boufounos, Member, IEEE, Alan V. Oppenheim, Life Fellow, IEEE, and Vivek K Goyal, Senior Member, IEEE
}

\begin{abstract}
In a variety of signal processing and communications contexts, erasures occur inadvertently or can be intentionally introduced as part of a data reduction strategy. This paper discusses causal compensation for erasures in frame representations of signals. The approach described assumes linear synthesis of the signal using a prespecified frame but no specific generation mechanism for the coefficients. Under this assumption, it is demonstrated that erasures can be compensated for using low-complexity causal systems. If the transmitter is aware of the occurrence of the erasure, an optimal compensation is to project the erasure error to the remaining coefficients. It is demonstrated that the same compensation can be executed using a transmitter/receiver combination in which the transmitter is not aware of the erasure occurrence. The transmitter precompensates using projections, as if assuming erasures will occur. The receiver undoes the compensation for the coefficients that have not been erased, thus maintaining the compensation only of the erased coefficients. The stability of the resulting systems is explored, and stability conditions are derived. It is shown that stability for any erasure pattern can be enforced by optimizing a constrained quadratic program at the system design stage. The paper concludes with examples and simulations that verify the theoretical results and illustrate key issues in the algorithms.
\end{abstract}

Index Terms-Erasures, frames, overcomplete signal representations.

\section{INTRODUCTION}

$\mathbf{I}$ $\mathrm{N}$ a variety of signal processing and communications contexts, erasures occur inadvertently or can be intentionally introduced as part of a data reduction strategy. Frame representations are generalizations of basis representations providing redundancy and, therefore, robustness to signal degradation such as noise, quantization, and erasures. This paper explores the use of projections to compensate for erasures in frame representations.

\section{A. Background}

A finite frame represents a vector $\mathbf{x}$ in a space $\mathcal{W}$ of finite dimension $N$ using the synthesis equation

$$
\mathbf{x}=\sum_{k=1}^{M} a_{k} \mathbf{f}_{k}
$$

Manuscript received July 24, 2006; revised July 5, 2007. This work was supported in part by the Texas Instruments Leadership University Consortium Program, BAE Systems Inc., and by MIT Lincoln Laboratory. The associate editor coordinating the review of this manuscript and approving it for publication was Dr. Zoran Cvetkovic.

P. Boufounos was with the Massachusetts Institute of Technology, Cambridge, MA 02139 USA. He is now with the Electrical and Computer Engineering Department, Rice University, Houston, TX 77251 USA (e-mail: petrosb@rice.edu).

A. V. Oppenheim and V. K. Goyal are with the Massachusetts Institute of Technology, Cambridge, MA 02139 USA (e-mail: avo@mit.edu; vgoyal@mit. edu).

Digital Object Identifier 10.1109/TSP.2007.908963 in which the $a_{k}$ are the representation coefficients, and the synthesis frame vectors $\left\{\mathbf{f}_{k}, k=1, \ldots, M\right\}$ span the space $\mathcal{W}$. This condition requires $M \geq N$. The ratio $r=M / N$ denotes the redundancy of the frame. For infinite-dimensional spaces, the definition above is extended to ensure the sum converges for all $\mathbf{x}$ with finite magnitude.

Frames are also used as analysis vector sets to determine a set of representation coefficients using inner products

$$
a_{k}=\left\langle\mathbf{x}, \underline{\mathbf{f}}_{k}\right\rangle
$$

in which the analysis frame vectors $\left\{\underline{\mathbf{f}}_{k}, k=1, \ldots, M\right\}$ also span $\mathcal{W}$. These coefficients can subsequently be used to reconstruct $\mathbf{x}$ using, for example, an appropriate synthesis set. Details on frame representations and the relationships of the analysis and synthesis vector sets can be found in a variety of texts such as [1] and [2].

The coefficients $a_{k}$ that represent a vector $\mathbf{x}$ using a prespecified synthesis frame $\left\{\mathbf{f}_{k}\right\}$ and the synthesis (1) can be determined in a variety of ways (for some examples, see [3]-[5] and references within). Similarly, the coefficients $a_{k}$ of a vector analyzed using the analysis frame and (2) can be used in a variety of ways to synthesize the vector. For example, it is not necessary to use all the coefficients to reconstruct the vector. A subset of the coefficients is sufficient to represent the vector as long as the corresponding frame vectors still span the space. In this case, perfect reconstruction is possible, making the representation robust to erasures during transmission [5].

Most of the previous work in erasures on frame representations assumes that $\mathbf{x}$ is represented using inner products with a fixed analysis frame. Under this assumption, the synthesis is modified to reconstruct the original signal despite erasures of certain components. For example, linear reconstruction can be performed using a recomputed synthesis frame and (1) [5], [6]. Alternatively, the nonerased coefficients can be used to recompute the erased in order to fill in the coefficient stream. The vector is linearly synthesized using the recovered stream and the original synthesis frame [7]. However, neither approach is possible without assuming an expansion using (2).

In this paper, rather than assuming that the vector is analyzed using the analysis (2), we make no assumptions on how the representation coefficients $a_{k}$ are generated. We only assume that the synthesis is performed using a prespecified synthesis frame and the synthesis sum of (1). The representation coefficients may be generated in a variety of ways, including but not limited to the analysis equation, matching pursuit [3], or basis pursuit [4]. Without assuming anything about the origin of the coefficients, it is not possible to fill in for the missing ones or appropriately modify the synthesis frame at the receiver. 
In principle, it is possible to synthesize $\mathbf{x}$ at the transmitter using the synthesis frame and the synthesis sum of (1). Subsequently, a frame representation can be recomputed using any analysis frame $\left\{\phi_{k}\right\}$ with the same redundancy and transmit these coefficients instead. The receiver receives some of the recomputed coefficients and synthesizes $\mathbf{x}$ using the dual of $\left\{\phi_{k}\right\}$ given the erasure pattern, as discussed in [5]-[7]. This approach, however, requires significant computation and knowledge of most of the erasure pattern either at the transmitter or the receiver, which can generate significant delays in the reconstruction of the signal.

The algorithms described in this paper, instead, modify the representation coefficients using orthogonal projections at the transmitter to compensate for an erasure. This assumes that the transmitter is aware that an erasure occurs, which is the first case considered. We also consider the case in which a transmitter encodes the coefficients such that only the receiver is aware that an erasure occurs. In this case, the transmitter modifies the frame representation assuming the erasures will occur, and the receiver undoes the changes if the erasures do not occur. The input-output behavior of the transmitter/receiver pair is identical to the input-output behavior of a transmitter which is aware of the erasure occurrence.

One advantage of using this approach is that the complete erasure pattern does not need to be known in advance. Furthermore, the representation coefficients may be generated in a variety of ways and it is not necessary to synthesize and reanalyze the signal $\mathbf{x}$ at the transmitter or the receiver. The drawback is that the causality and stability constraints imposed in part of this development often allow only for partial compensation of the error. The approach described here is more appropriate for large or infinite frame representations and streaming conditions, in which delay or computational complexity is important. This method is not well suited to applications using small finite frames, in which delay is not an issue. Furthermore, the transmitter described in Section III-B can potentially lead to an increase in the transmitted signal power, which might be an issue, depending on the application.

Frame designs that are robust to erasures and quantization have been extensively studied in the literature for various classes of frames, synthesis assumptions, and optimality criteria (for some examples, see [5], [6], [8]-[15]). Similar to the existing work on algorithms mentioned above, and contrary to the approach presented in this paper, most of this work assumes the frame is used to perform analysis using inner products instead of the linear synthesis equation. Some design principles carry over to the compensation methods presented in this paper. Careful examination of the frame design in the context of the algorithms presented here is an interesting topic beyond the scope of this paper. Furthermore, in several applications in which these algorithms are applicable the frame is not designed but predetermined by the problem.

The use of projections to compensate for erasures is similar to their use in [16] to extend quantization noise shaping to arbitrary frame expansions. However, in [16], the quantization error is known at the transmitter-not necessarily the case with erasure errors. The use of redundancy to compensate for erasures assuming a fixed reconstruction method has also been considered in a different context in [17]. In that work the error is again known at the transmitter and only the case of LTI reconstruction filters is considered. The problem is formulated and solved as a constrained optimization.

\section{B. Applications}

The potential applications of this approach are several. One area of applications is in faulty D/A converters that fail to output some of the coefficients in the reconstruction, replacing the corresponding coefficients by zero, as described in [17]. LCD displays with broken pixels are one example of this application. The causality constraints imposed in the second half of this development make the algorithms applicable to streaming D/A devices that drop samples randomly in time due to hardware failures.

Another application is in distributed sensor networks, in which each sensor records measurements from a field and sequentially transmits them to a central processing node using a time-division multiplexing algorithm. The central node uses the frame synthesis equation to combine these measurements for further processing. Due to power or communication link conditions a sensor might not be able to communicate to the central node at some point in time to transmit a measurement but is able to transmit the measurement to the nearby sensors that have not transmitted their own measurements yet. The nearby sensors modify their own measurements, as described in this paper, and subsequently transmit the modified measurements such that the erasure is causally compensated for in a distributed manner before the reconstruction. Several suitable scenarios are described in [18].

It is also possible that a data acquisition device introduces erasures intentionally, for example using randomized sampling [19], [20], to reduce the average output rate of samples, possibly at the cost of some error. The algorithms described in this paper can be used to reduce or eliminate the error due to the erasures, thus improving the data acquisition performance, providing an alternative to spectrally shaping the random sampling pattern as described in [20].

It should be noted that this paper does not provide a detailed solution to any of the above applications. These serve only as a motivation to address the particular problem. This paper introduces and discusses two algorithms for the compensation of erasures using projections, abstracted from the application details. Application of the algorithms presented in this work to particular applications requires further analysis specific to the application, which includes assumptions on the application constraints, the frame design, and the erasure characteristics.

\section{Outline}

The next section states the problem and establishes the notation. It is shown that the optimal solution is the orthogonal projection of the erasure error to the span of the remaining synthesis vectors, and some properties of sequential compensations are proved. A causal implementation is proposed in Section III-A, assuming the transmitter is aware of the erasure. Section III-B presents a system in which the transmitter precompensates for the erasure and the receiver undoes the compensation if the erasure does not occur. The stability of the algorithms is discussed in Section III-C. Some key issues for practical implementations are illustrated in the examples presented in Section IV. 
Appendix A proves the input-output equivalence of the two algorithms presented in Section III-A and B, and Appendix B proves some of the stability results discussed in Section III-C

\section{ERASURe COMPENSATION Using Projections}

After stating the problem and establishing notation, this section examines the compensation for a single erasure. In Section II-C the results are extended to the compensation of multiple erasures, and properties of sequential compensations are considered.

\section{A. Problem Statement}

We consider the synthesis of a vector $\mathbf{x}$ using (1), in which we make no assumptions on how the representation coefficients $\left\{a_{k}\right\}$ originate. The $\left\{a_{k}\right\}$ might even be data to be processed using the synthesis sum (1), such as a discrete-time signal to be filtered, not originating from the analysis of $\mathbf{x}$.

The coefficients $\left\{a_{k}\right\}$ are used to synthesize the signal using the prespecified synthesis frame $\left\{\mathbf{f}_{k}\right\}$, subject to erasures known at the transmitter or the receiver. We model erasures as replacement of the corresponding $a_{k}$ with 0 , i.e. removal of the corresponding term $a_{k} \mathbf{f}_{k}$ from the summation in (1). Since the analysis method is not known, the goal is to compensate for the erasure as much as possible using the remaining nonerased coefficients.

In Section III-A, we assume that the transmitter anticipates an erasure and knows the value of the erased coefficient. Assuming coefficient $a_{i}$ is erased, the transmitter is constrained to only replace the coefficients $\left\{a_{k} \mid k \in S_{i}\right\}$ with $\left\{\hat{a}_{k} \mid k \in S_{i}\right\}$ in order to compensate for the erasure, where $S_{i}=\left\{k_{1}, \ldots, k_{P}\right\}$ denotes the set of coefficient indices used for the compensation of $a_{i}$. The reconstruction is performed using (1) with the updated coefficients:

$$
\hat{\mathbf{x}}=\sum_{k \in S_{i}} \hat{a}_{k} \mathbf{f}_{k}+\sum_{k \notin S_{i}, k \neq i} a_{k} \mathbf{f}_{k}
$$

such that $\hat{\mathbf{x}}$ minimizes the magnitude of the error $\mathcal{E}=\mathbf{x}-\hat{\mathbf{x}}$.

\section{B. Compensation of a Single Erasure}

The error due to the erasure of a single coefficient $a_{i}$ and its subsequent compensation using the coefficients $\left\{a_{k} \mid k \in S_{i}\right\}$ can be rewritten using the synthesis sum

$$
\mathcal{E}=a_{i} \mathbf{f}_{i}+\sum_{k \in S_{i}}\left(a_{k}-\hat{a}_{k}\right) \mathbf{f}_{k} .
$$

The span of $\left\{\mathbf{f}_{k} \mid k \in S_{i}\right\}$ is henceforth denoted using $\mathcal{W}_{i}$. The error magnitude is minimized if the sum $\sum_{k \in S_{i}}\left(a_{k}-\hat{a}_{k}\right) \mathbf{f}_{k}$ is the orthogonal projection of $-a_{i} \mathbf{f}_{i}$ onto $\mathcal{W}_{i}$.

To compute the projection we define the projection coefficients $c_{i, k}$ such that they satisfy

$$
\mathcal{P}_{\mathcal{W}_{i}}\left(\mathbf{f}_{i}\right)=\sum_{k \in S_{i}} c_{i, k} \mathbf{f}_{k}
$$

in which $\mathcal{P}_{\mathcal{W}_{i}}\left(\mathbf{f}_{i}\right)$ is the projection of $\mathbf{f}_{i}$ onto $\mathcal{W}_{i}$. By taking inner products on both sides with all $\mathbf{f}_{k}$, it follows that the projection coefficients satisfy:

$$
\left[\begin{array}{ccc}
R_{k_{1}, k_{1}} & \cdots & R_{k_{1}, k_{P}} \\
\vdots & \ddots & \vdots \\
R_{k_{P}, k_{1}} & \cdots & R_{k_{P}, k_{P}}
\end{array}\right]\left[\begin{array}{c}
c_{i, k_{1}} \\
\vdots \\
c_{i, k_{P}}
\end{array}\right]=\left[\begin{array}{c}
R_{i, k_{1}} \\
\vdots \\
R_{i, k_{P}}
\end{array}\right] \Leftrightarrow \mathbf{R c}=\boldsymbol{\rho}
$$

in which $R_{k, l}=\left\langle\mathbf{f}_{k}, \mathbf{f}_{l}\right\rangle$ is the frame autocorrelation function.

The projection coefficients are used to optimally compensate for the erasure $a_{i}$ by updating each of the $a_{k}$ to

$$
\hat{a}_{k}=a_{k}+a_{i} c_{i, k}, \quad \text { for all } k \in S_{i} .
$$

Consequently

$$
\begin{aligned}
\mathcal{E} & =a_{i} \mathbf{f}_{i}-a_{i} \sum_{k \in S_{i}} c_{i, k} \mathbf{f}_{k} \\
& =a_{i}\left(\mathbf{f}_{i}-\mathcal{P}_{\mathcal{W}_{i}}\left(\mathbf{f}_{i}\right)\right) \\
& =a_{i} \epsilon_{i} \mathbf{r}_{i}
\end{aligned}
$$

in which $\epsilon_{i}$ and $\mathbf{r}_{i}$ are the error coefficient and the residual direction, defined as:

$$
\begin{aligned}
\epsilon_{i} & =\left\|\mathbf{f}_{i}-\mathcal{P}_{\mathcal{W}_{i}}\left(\mathbf{f}_{i}\right)\right\|, \text { and } \\
\mathbf{r}_{i} & =\frac{\mathbf{f}_{i}-\mathcal{P}_{\mathcal{W}_{i}}\left(\mathbf{f}_{i}\right)}{\left\|\mathbf{f}_{i}-\mathcal{P}_{\mathcal{W}_{i}}\left(\mathbf{f}_{i}\right)\right\|}
\end{aligned}
$$

respectively. The error coefficient, $\epsilon_{i}$, ranging from 0 to $\left\|\mathbf{f}_{i}\right\|$, quantifies how much of the erasure is compensated for, 0 being perfect compensation and $\left\|\mathbf{f}_{i}\right\|$ being no compensation. The residual direction $\mathbf{r}_{i}$ is a unit norm vector in the direction of the error.

The residual error after each compensation can be further reduced using additional frame coefficients for the compensation. Adding more coefficient indices in the set $S_{i}$, and, therefore, using additional frame vectors for the compensation of the error, can enlarge the space $\mathcal{W}_{i}$ and decrease the error magnitude. Using more coefficients for the compensation decreases the error magnitude only if the vector $\mathbf{f}_{l}$ added to the compensation set is not orthogonal to the residual direction $\mathbf{r}_{i}$ of the error before the vector is added.

Satisfying (6) is equivalent to computing the frame expansion of $\mathbf{f}_{i}$ using $\left\{\mathbf{f}_{k} \mid k \in S_{i}\right\}$ as a synthesis frame. If the frame vectors $\left\{\mathbf{f}_{k} \mid k \in S_{i}\right\}$ are linearly dependent, the solution to (6) is not unique. All the possible solutions are optimal in terms of minimizing the error magnitude, given the constraint that only coefficients $\left\{a_{k} \mid k \in S_{i}\right\}$ can be modified. If the vector $a_{i} \mathbf{f}_{i}$ which is being compensated is in the span of the vectors $\left\{\mathbf{f}_{k} \mid k \in S_{i}\right\}$ used for the compensation (i.e., $\mathbf{f}_{i} \in \mathcal{W}_{i}$ ), then the erasure is fully compensated for. In this case the error is zero, and we call the compensation complete.

\section{Compensation of Multiple Coefficients}

In the development above we assume only one erasure. Projection-based compensation can be generalized to the sequential erasure of multiple expansion coefficients, allowing a subset of the remaining coefficients for each compensation. If multiple 
coefficients are erased and none of the erased coefficients have been previously used to compensate for another erasure, the optimal compensation is a straightforward application of the results of the previous section. If however, a coefficient used to compensate for an erasure is then erased and compensated for, the method is only locally optimal. This section examines some of the properties and some conditions under which the compensation of multiple coefficients is also globally optimal subject to the system design constraints.

We assume that the sets $S_{i}$ of coefficients used to compensate each of the erasures are part of the system design. We further assume that once a coefficient has been erased and compensated for, it is not used to compensate for subsequent erasures. Under these assumptions four properties of the compensation are derived. In formulating these, the term optimal is used if the compensation minimizes the error given the constraints and the term complete is used if the error after the compensation is exactly zero. These properties are described in this section.

1) Compensation of the Error is Equivalent to Projection of the Data: Consider the vector $\mathbf{y}$ that can be synthesized from the erased coefficient $a_{i}$ and the coefficients to be modified $\left\{a_{k} \mid k \in S_{i}\right\}$. Compensating for the erasure is equivalent to projecting $\mathbf{y}$ to the space $\mathcal{W}_{i}$, spanned by the frame vectors corresponding to the coefficients to be modified. Specifically, if

$$
\mathbf{y}=a_{i} \mathbf{f}_{i}+\sum_{k \in S_{i}} a_{k} \mathbf{f}_{k}
$$

then

$$
\sum_{k \in S_{i}} \hat{a}_{k} \mathbf{f}_{k}=\mathcal{P}_{\mathcal{W}_{i}}(\mathbf{y})
$$

This also implies that the error after the compensation $a_{i} \epsilon_{i} \mathbf{r}_{i}$, and consequently the residual direction $\mathbf{r}_{i}$, is orthogonal to all the frame vectors used for compensation.

2) Superposition: Using the linearity of projections it follows that:

$$
\mathcal{P}_{\mathcal{W}_{i}}\left(a_{i} \mathbf{f}_{i}+a_{j} \mathbf{f}_{j}\right)=\mathcal{P}_{\mathcal{W}_{i}}\left(a_{i} \mathbf{f}_{i}\right)+\mathcal{P}_{\mathcal{W}_{i}}\left(a_{j} \mathbf{f}_{j}\right)
$$

Furthermore, if $S_{i}=S_{j}$ then $\mathcal{W}_{i}=\mathcal{W}_{j}$. Thus, if the set of coefficients $S_{i}=S_{j}$ is used to separately compensate for the erasure of two different coefficients $a_{i}$ and $a_{j}$, then the superposition of the individual compensations produces the same error as the erasure of a single vector $a_{i} \mathbf{f}_{i}+a_{j} \mathbf{f}_{j}$ followed by compensation using the same set of coefficients $S_{i}$.

3) Sequential Superposition: If $\mathcal{W}_{j} \subseteq \mathcal{W}_{i}$ then

$$
\mathcal{P}_{\mathcal{W}_{j}}\left(\mathcal{P}_{\mathcal{W}_{i}}(\mathbf{y})\right)=\mathcal{P}_{\mathcal{W}_{j}}(\mathbf{y}) \text {. }
$$

Furthermore, if $S_{j} \subseteq S_{i}$ then $\mathcal{W}_{j} \subseteq \mathcal{W}_{i}$. Consider the case in which one of the updated coefficients $\hat{a}_{j}, j \in S_{i}$, used in the compensation of $a_{i}$, is subsequently erased and optimally compensated for using the remaining coefficients in $S_{i}$. Using properties 1 and 2 , this becomes equivalent to the following projection sequence of the data:

$$
\sum_{k \in S_{j}} \check{a}_{k} \mathbf{f}_{k}=\mathcal{P}_{\mathcal{W}_{j}}\left(\mathcal{P}_{\mathcal{W}_{i}}\left(a_{i} \mathbf{f}_{i}+\sum_{k \in S_{i}} a_{k} \mathbf{f}_{k}\right)\right)
$$

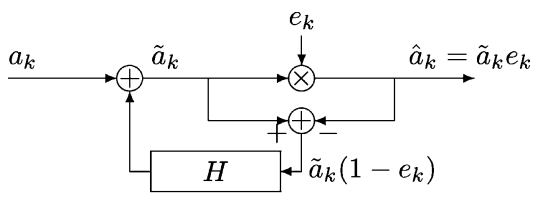

Fig. 1. Erasure-aware transmitter projecting erasure errors.

in which $S_{j}=\left\{k \neq j \mid k \in S_{i}\right\}$ contains all the elements of $S_{i}$ except for $j$, and $\left\{\breve{a}_{k} \mid k \in S_{j}\right\}$ is the set of the updated coefficients after both erasures of $a_{i}$ and of $\hat{a}_{j}$ have been compensated. Therefore, this is equivalent to optimally compensating both $a_{i}$ and $a_{j}$ using the coefficients in $S_{j}$.

4) Sequential Complete Compensation: If an $a_{j}, j \in S_{i}$ used in the compensation of $a_{i}$ is subsequently erased but completely compensated using the set $S_{j}$, the compensation of $a_{i}$ is still optimal since the incremental error of the second compensation is zero.

If the compensation of $a_{i}$ was complete, the total error after both compensations is zero. In this case

$a_{i} \mathbf{f}_{i}+a_{j} \mathbf{f}_{j}+\sum_{k \in S_{i, j}} a_{k} \mathbf{f}_{k}=\mathcal{P}_{\mathcal{W}_{i, j}}\left(a_{i} \mathbf{f}_{i}+a_{j} \mathbf{f}_{j}+\sum_{k \in S_{i, j}} a_{k} \mathbf{f}_{k}\right)$

in which $S_{i, j}=\left\{k \neq j \mid k \in\left(S_{i} \cup S_{j}\right)\right\}$ is the combined set of indices used to compensate for the erasure of $a_{i}$ and $a_{j} . \mathcal{W}_{i, j}$ is the space spanned by the corresponding frame vectors. Therefore, using property 1 , the sequential complete compensation in this case is equivalent to optimally and completely compensating the erasure of both $a_{i}$ and $a_{j}$ using the set $S_{i, j}$.

\section{CAusal COMPEnsation}

For the remainder of this paper, we assume the coefficients are transmitted in sequence, indexed by $k$ in (1). We focus on causal compensation in which only a finite number of coefficients subsequent to the erasure are used for compensation. In this section we examine the causal compensation of coefficient erasures using a transmitter aware of the erasure occurrence. We also develop a transmitter/receiver pair that implements the same causal compensation method but requires only the receiver to be aware of the erasure occurrence.

\section{A. Transmitter-Aware Compensation}

If the transmitter is aware of the erasure occurrence, the projections are straightforward to implement using the system in Fig. 1. In the figure, $e_{k}$ denotes a binary sequence of ones and zeros, which multiplicatively implements the erasures, and $H$ is a linear system that performs the compensation. To compensate for the erasure, the system uses only the $P$ coefficients subsequent to the erased one, which implies that the set $S_{k}$ is equal to $S_{k}=\{k+1, \ldots, k+P\}$. The system resembles Sigma-Delta noise shaping systems; projection-based compensation of errors is introduced in [16] to extend Sigma-Delta noise shaping to arbitrary frames.

For clarity of the exposition, we first develop the algorithm for a shift-invariant frame. Such a frame has autocorrelation that is a function only of the index difference, i.e., satisfies $R_{k, l}=$ 
$R_{k-l, 0} \equiv R_{k-l}$. In this case, $c_{k, k+i}=c_{0, i} \equiv c_{i}$, and the linear feedback system $H$ in Fig. 1 is time-invariant with impulse response

$$
h_{n}=\sum_{m=1}^{P} c_{m} \delta_{n-m} .
$$

The compensation is optimal if the erasures are sufficiently rare such that there is only one erasure within $P$ coefficients, or if $P$ is such that the erasure compensation is complete. Otherwise it is only a locally optimal strategy which minimizes the incremental error after an erasure has occurred, subject to the design constraints.

For arbitrary, shift-varying frames, the linear feedback system $H$ is time varying with coefficients that satisfy (6) at the corresponding time point. Specifically, $y_{k}$, the output of $H$, is

$$
y_{k}=\sum_{m=1}^{P} c_{k-m, k} x_{k-m}
$$

in which $x_{k}=a_{k}\left(1-e_{k}\right)$ is the input.

The input and the output of the transmitter satisfy

$$
\begin{aligned}
\tilde{a}_{k}= & \sum_{m=1}^{P}\left(1-e_{k-m}\right) c_{k-m, k} \tilde{a}_{k-m}+a_{k} \\
\hat{a}_{k}= & \tilde{a}_{k} e_{k} \\
\Rightarrow a_{k}= & \hat{a}_{k}+\left(1-e_{k}\right) \tilde{a}_{k} \\
& -\sum_{m=1}^{P}\left(1-e_{k-m}\right) c_{k-m, k} \tilde{a}_{k-m} .
\end{aligned}
$$

This is a recursive algorithm. Although an erasure of $a_{k}$ is compensated using only the next $P$ coefficients, one of these next coefficients $a_{l}, l \leq k+P$ might be subsequently erased. In this case, the algorithm compensates for the erasure of the modified coefficient $\hat{a}_{l}$, i.e., for the erasure of the original data in $a_{l}$ and for the additive part due to the compensation of $a_{k}$. Thus, the feedback loop is potentially unstable. In Section III-C, we explore some stability conditions for this feedback loop.

\section{B. Precompensation With Correction}

In many systems, particularly in streaming applications, the transmitter is not aware of the erasure occurrence. In such situations it is possible to preproject the error at the transmitter side, assuming an erasure will occur. If the erasure does not occur, the receiver undoes the compensation. It should be emphasized that the algorithm described in this section has identical input-output behavior to the one described in Section III-A. Therefore, all the performance analysis for that algorithm applies to this one as well. The input-output equivalence is proven in Appendix A.

To pre-compensate for the erasure, the transmitter at step $k$ updates the subsequent coefficients $a_{k+1}, \ldots, a_{k+p}$ to

$$
a_{k+m}^{\prime}=a_{k+m}+c_{k, k+m} a_{k}^{\prime}
$$

where the $c_{k, k+m}, m=1, \ldots, P$ satisfy (6). The $a_{k}^{\prime}$ used for the update is the coefficient as updated from all the previous iterations of the algorithm, not the original coefficient of the expansion, making the transmitter a recursive system. Depending on the frame, the transmitter might be unstable. This issue

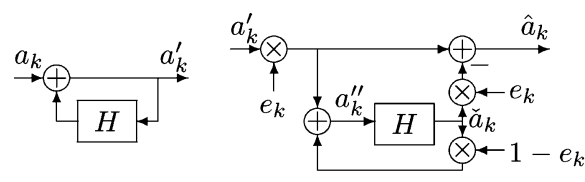

(a)

(b)

Fig. 2. Transmitter and receiver structure projecting erasure errors. Only the receiver is aware of the erasure. (a) Transmitter. (b) Receiver.

is separate from the stability of the compensation algorithm, raised previously. Stability of this transmitter is also discussed in Section III-C.

If an erasure does not occur the receiver at time step $k$ receives coefficient $a_{k}^{\prime}$ and sets $a_{k}^{\prime \prime}=a_{k}^{\prime}$. Otherwise, it sets

$$
\begin{aligned}
a_{k}^{\prime \prime} & =\check{a}_{k} \\
\text { with } \quad \check{a}_{k} & =\sum_{m=1}^{P} c_{k-m, k} a_{k-m}^{\prime \prime}
\end{aligned}
$$

which is the part of $a_{k}^{\prime}$ from (24) that is due to the projection of the nonerased coefficients. An erasure also erases the components of $a_{k}^{\prime}$ due to the projection of the previously received coefficients. The variables $\breve{a}_{k}$ in (26) ensure that these components can be removed from the subsequently received coefficients even when $a_{k}^{\prime}$ has not been received.

The receiver output $\hat{a}_{k}$ is conditional on whether an erasure has occurred or not

$$
\hat{a}_{k}=\left(a_{k}^{\prime}-\check{a}_{k}\right) e_{k}= \begin{cases}0, & \text { if } e_{k}=0 \\ a_{k}^{\prime}-\check{a}_{k}, & \text { otherwise. }\end{cases}
$$

This removes the projection of the previously received coefficients from $a_{k}^{\prime}$.

The reconstruction in (27) undoes the recursive effects of (24) and ensures that the projection only affects the $P$ coefficients subsequent to the erasure. The system is depicted in Fig. 2, in which $e_{k}$, the sequence of ones and zeros denoting the erasures, is the same in all three locations in the figure. The systems $H$ are the same as in Fig. 1. In Appendix A, it is shown that the two systems are input-output equivalent.

In several applications, such as packetized transmissions, frame expansions are used for transmission of blocks of coefficients. In such cases, the systems described can be modified using property 2 in Section II-C to accommodate block erasures by projecting the whole vector represented by the transmitted block to the subsequent coefficients.

\section{Compensation Stability}

The systems in Figs. 1 and 2 can potentially be unstable, depending on the frame and the erasure pattern. This section examines some aspects of the instability and provides a necessary condition and a sufficient condition for the systems to be stable. In this discussion, stability refers to bounded-input-boundedoutput (BIBO) stability of the systems. All stability conditions presented in this section can be summarized using the system in Fig. 3 for appropriate choice of the linear system $H_{s}$.

In examining the system robustness, a separate issue is the performance subject to external disturbances such as parameter mismatches, quantization, and different initial conditions. 


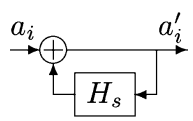

Fig. 3. System summarizing the stability conditions for the algorithms. Depending on the stability condition considered, the system in the figure should be stable with $H_{s}$ satisfying one of the input-output relations in (34), (36), or (33).

This issue, while important and interesting, is beyond the scope of this paper. The results in this section can only provide a guarantee that the design remains stable subject to these disturbances.

1) Stability of the Compensation Algorithm: The evolution of the system variables is determined by (21). By taking the expected value of both sides, this becomes

$$
\tilde{\mu}_{k}=\sum_{m=1}^{P} q c_{k-m, k} \tilde{\mu}_{k-m}+\mu_{k}
$$

in which $\mu_{k}=E\left\{a_{k}\right\}, \tilde{\mu}_{k}=E\left\{\tilde{a}_{k}\right\}$, and $q=P\left(e_{k}=0\right)$ is the probability of erasures. Therefore, the compensation algorithm is stable in the mean if and only if the time varying system in (28) is stable. For a shift-invariant frame this is equivalent to the LTI system $H(z)=1 /\left(1-\sum_{m=1}^{P} q c_{m} z^{-m}\right)$ being stable. Stability in the mean is a necessary but not sufficient condition for system stability since the variance can diverge.

A sufficient condition for stability can be derived using the triangle inequality to determine an upper bound for the magnitude of the coefficients

$$
\left|\tilde{a}_{k}\right| \leq \sum_{m=1}^{P}\left|c_{k-m, k}\right| \cdot\left|\tilde{a}_{k-m}\right|+\left|a_{k}\right| .
$$

Therefore, assuming a bounded input $\left|a_{k}\right|$, the stability of the algorithm is guaranteed for all $q$ if the system in (29) is stable.

It is further shown in Appendix B that if the erasure process is independent of the frame expansion coefficients $a_{k}$, the second moment of the compensated coefficients has upper bound

$$
\sqrt{E\left\{\tilde{a}_{k}^{2}\right\}} \leq \sum_{m=1}^{P} \sqrt{q}\left|c_{k-m, k}\right| \cdot \sqrt{E\left\{\tilde{a}_{k-m}^{2}\right\}}+\sqrt{E\left\{a_{k}^{2}\right\}}
$$

which implies that if the system in (30) is stable, then the variance is bounded, and, therefore, the coefficients are bounded with probability one.

For shift-invariant frames, the conditions in (29) and (30) are equivalent to the systems $H(z)=1 /\left(1-\sum_{m=1}^{P}\left|c_{m}\right| z^{-m}\right)$ and $H(z)=1 /\left(1-\sum_{m=1}^{P} \sqrt{q}\left|c_{m}\right| z^{-m}\right)$ being stable, respectively. These are only sufficient conditions for stability.

In Appendix B, it is also shown that BIBO stability of the systems in (29), and (30) can be guaranteed if the sum of the coefficient magnitudes is less than 1, i.e.

$$
\begin{aligned}
& \sum_{m=1}^{P}\left|c_{k-m, k}\right|<1, \text { and } \\
& \sum_{m=1}^{P}\left|c_{k-m, k}\right|<\eta=1 / \sqrt{q}
\end{aligned}
$$

respectively.
It follows that if a system satisfies (32) for probability of erasure $q_{o}$, it also satisfies it for any $q \leq q_{o}$. First-order systems always have $\left|c_{k-1, k}\right| \leq 1$, which implies that first order optimal compensation algorithms are always stable.

Summarizing in terms of the system in Fig. 3, if the system is stable for $H_{s}$ that satisfies

$$
y_{k}=\sum_{m=1}^{P} \sqrt{q}\left|c_{k-m, k}\right| x_{k-m}
$$

in which $x_{k}$ and $y_{k}$ are the input and output to $H_{s}$, respectively, then the compensation algorithm is stable for any erasure pattern with probability of erasure less than $q$. Furthermore, the compensation algorithm is stable at probability of erasure $q$ only if the system in Fig. 3 is stable for $H_{s}$ that satisfies

$$
y_{k}=\sum_{m=1}^{P} q c_{k-m, k} x_{k-m}
$$

2) Transmitter and Receiver Stability: The analysis above considers the stability of the compensation algorithm. However, the stability of the transmitter and the receiver in Fig. 2 as independent systems is a separate issue. Even if the combined system is input-output equivalent to the system in Fig. 1, the combined system might exhibit internal instabilities which can be triggered, for example, by mismatched initial conditions or other disturbances. The conditions derived here permit the separation of the algorithm into two systems that are stable on their own. However, the effect of external disturbances other than erasures on the performance is not considered in this paper.

The output $a_{k}^{\prime}$ of the transmitter in Fig. 2(a) follows the same dynamics as the mean $\tilde{m}_{k}$ in (28) with $q=1$. Therefore, the transmitter is a stable system if and only if the compensation algorithm is stable in the mean for $q=1$. BIBO stability of the compensation algorithm for $q=1$ guarantees stability in the mean for $q=1$, which implies a stable transmitter.

The receiver variable $\breve{a}_{k}$ follows the same dynamics as the overall compensation algorithm in (21):

$$
\check{a}_{k}=\sum_{m=1}^{P}\left(1-e_{k-m}\right) c_{k-m, k} \check{a}_{k-m}+\sum_{m=1}^{P} e_{k-m} c_{k-m, k} a_{k-m}^{\prime}
$$

in which $e_{k} a_{k}^{\prime}$ is the receiver input, and the sum $\sum_{m=1}^{P} c_{k-m, k} a_{k-m}^{\prime} e_{k-m}$ is the receiver input through a finite response system. If the compensation algorithm is stable at a certain probability of erasures, then (35) is also stable even if the second sum is replaced by an arbitrary input. Thus, stability of the compensation algorithm implies that the receiver is stable for any input, even if the input did not originate from the transmitter in Fig. 2(a).

In summary, the only additional condition for the system to be internally stable in a separate transmitter/receiver configuration is that the transmitter in Fig. 2(a) is stable. This is identical to the system in Fig. 3 for $H_{s}$ that satisfies the transmitter dynamics

$$
y_{k}=\sum_{m=1}^{P} c_{k-m, k} x_{k-m}
$$

in which $x_{k}$ and $y_{k}$ are the input and output to $H_{s}$, respectively. 


\section{Enforcing a Stable Design}

The solution to (6) might not provide coefficients that produce stable systems. In these cases, the projection coefficients should be such that they balance the optimality of the projection with the stability of the system. Although the condition in (31) is sufficient to guarantee stability in all situations, it is very severe, and not necessary, especially in conditions with low probability of erasures. It is often sufficient to enforce the constraint in (32) for the appropriate choice of $\eta=1 / \sqrt{q}$.

In either case, the constraints can be enforced by considering a constrained optimization approach. Specifically, the coefficients $c_{k-i, k}$ should be such that the incremental error magnitude is minimized subject to the constraint

$$
\min \left\|\mathbf{f}_{k}-\sum_{m=1}^{P} c_{k, k+m} \mathbf{f}_{k+m}\right\| \text {, s.t. } \sum_{m=1}^{P}\left|c_{k-m, k}\right|<\eta .
$$

The solution to this constrained quadratic program produces a system with guaranteed stability for all erasure patterns with probability of erasure less than $1 / \eta^{2}$, at the expense of an increase in the incremental error compared to the unconstrained approach. As $\eta$ grows the optimization eventually becomes a projection, satisfying (5).

It should be noted that in general the optimization is carried out once for each frame vector, while the constraint might be on the coefficients for the compensation of several frame vectors. Thus, it might be necessary to balance the optimization of competing cost functions subject to the constraints, for example by jointly optimizing a linearly weighted sum of the cost. The joint optimization might also generate a very large program if the frame is arbitrary. However, the optimization only needs to be performed once, at the system design stage. The optimization complexity does not affect the run-time complexity of the system. Furthermore, the frame might have enough structure to reduce the size of the problem. Some examples are shown in Section IV, but further discussion of this optimization process is beyond the scope of this paper.

\section{EXAMPLES AND SIMULATIONS}

This section presents two examples of the compensation algorithm described in the previous sections. The examples are chosen to demonstrate key features and tradeoffs in the algorithm.

In the first example, the signal is synthesized using the shiftinvariant frame implied by an LTI low-pass filter. This example demonstrates that the compensation performance improves with the order of the compensation and the redundancy of the frame. The frame is chosen to expose the tradeoff between stability and performance. A key feature of this example is that even when the algorithm is unstable for certain probabilities of erasure, the transmitter shown in Fig. 2(a) can be stable. Therefore, the implementation described in Section III-B is possible.

In the second example the synthesis is performed using an oversampled synthesis filterbank. This is an example in which extending the compensation beyond a certain order does not improve the compensation, and correspondingly the optimal compensation will be of finite order. The example illustrates that if the frame is not shift-invariant, it is necessary to compute more than one set of coefficients. Imposing, therefore, the stability constraint of (37) requires balancing the optimization of competing cost functions. The example further shows that for the same frame, higher order compensation might be stable and achieve better performance, even if a lower order compensation is unstable.

Although, the results presented assume i.i.d. erasures and white frame representation coefficients, the simulations were performed for a variety of erasure conditions, and frame coefficients spectra. Although the performance varies among different conditions, the simulations confirm the stability results and the performance improvements using the algorithm. Due to the local nature of the algorithm, the performance improvements are smaller in the case the erasures are correlated and greater in the case the erasures are anticorrelated. Unfortunately, in the interest of brevity, it is not possible to present more simulation results in this paper.

\section{A. Synthesis Using a Low-Pass Filter}

For a shift-invariant frame the autocorrelation $R_{k, l}$ is only a function of the index difference

$$
R_{k, l}=R_{k-l, 0} \equiv R_{k-l}=R_{m}
$$

In this case, assuming a fixed compensation order $P$ for each coefficient, the compensation coefficients are also shift-invariant, and (6) only needs to be solved once. The matrix $\mathbf{R}$ becomes a symmetric Toeplitz matrix, and the equation takes the special form of the Yule-Walker autocorrelation normal equations [21], [22]

$$
\left[\begin{array}{ccc}
R_{0} & \cdots & R_{P-1} \\
\vdots & \ddots & \vdots \\
R_{P-1} & \cdots & R_{0}
\end{array}\right]\left[\begin{array}{c}
c_{1} \\
\vdots \\
c_{P}
\end{array}\right]=\left[\begin{array}{c}
R_{1} \\
\vdots \\
R_{P}
\end{array}\right]
$$

in which $c_{m} \equiv c_{k, k+m}=c_{0, m}$. The special structure allows for an efficient solution using the Levinson-Durbin recursion [23], [24].

The convolution implied by LTI filtering can also be viewed as a linear synthesis equation in which the frame vectors are shifts of the time-reversed filter impulse response, and the input to the filter corresponds to the representation coefficients

$$
\begin{aligned}
& x[n]=\sum_{k} a_{k} h[n-k] \\
& \Leftrightarrow \mathbf{x}=\sum_{k} a_{k} \mathbf{f}_{k}
\end{aligned}
$$

with the frame autocorrelation given by the deterministic autocorrelation of the filter impulse response:

$$
R_{m}=\left\langle\mathbf{f}_{0}, \mathbf{f}_{m}\right\rangle=\sum_{n} h[n] h[n+m]
$$


(a) $r=4$

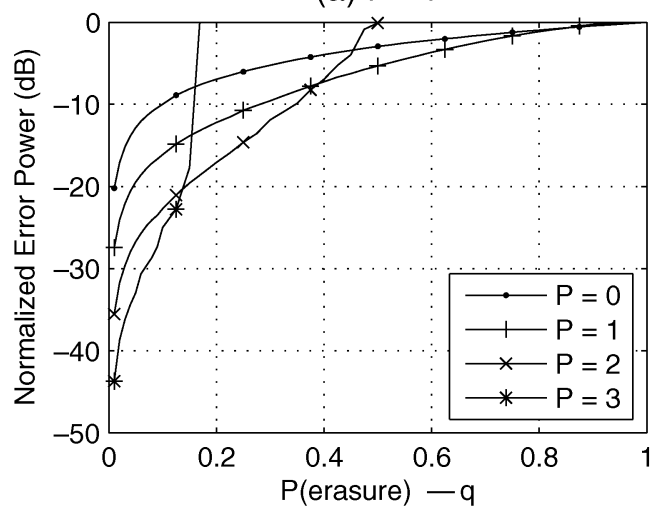

(c) $r=16, P=5$

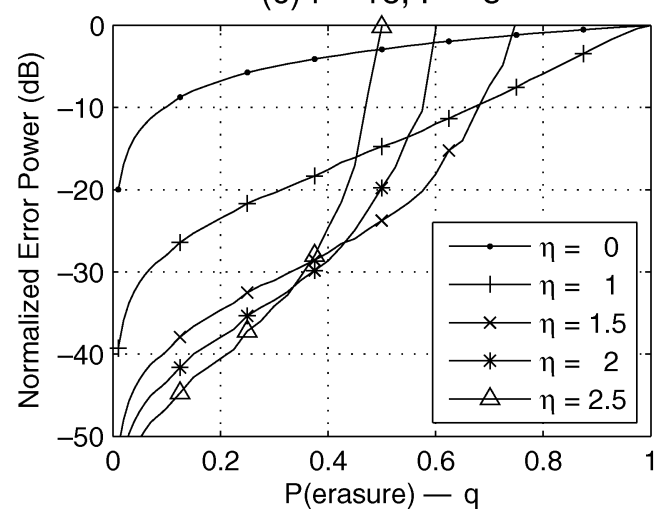

(b) $\mathrm{P}=2$

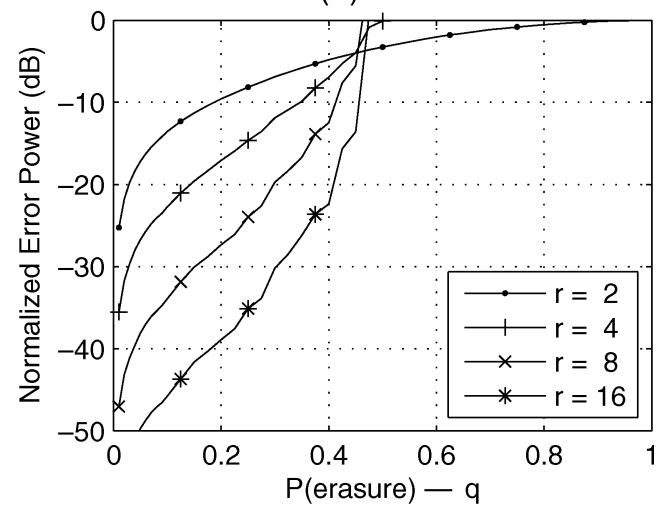

(d) $\eta=1.5, P=5$

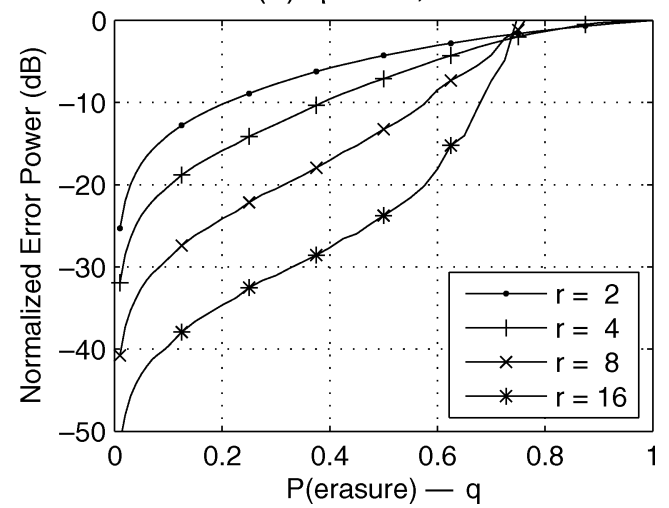

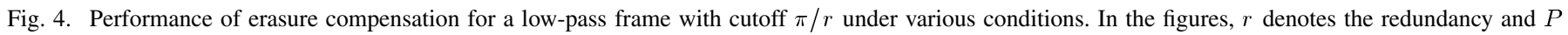

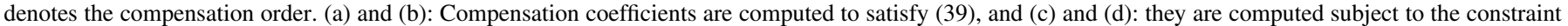
$\sum_{k=1}^{P}\left|c_{k}\right|<\eta$. (a) $r=4$. (b) $P=2$. (c) $r=16, P=5$. (d) $\eta=1.5, P=5$.

It follows that the system $H$ implementing the projections in Fig. 1 and 2 is an LTI system with transfer function

$$
H(z)=\sum_{m=1}^{P} c_{m} z^{-m}
$$

in which the $c_{m}$ are the projection coefficients from (39). Specifically, if $h[n]$ is a low-pass filter with cutoff frequency $\omega_{0}=$ $\pi / r$, the impulse response has the form of a $\operatorname{sinc}(\cdot)$ function, and (39) becomes

$$
\begin{array}{r}
{\left[\begin{array}{ccc}
\operatorname{sinc}(0 / r) & \cdots & \operatorname{sinc}((P-1) / r) \\
\vdots & \ddots & \vdots \\
\operatorname{sinc}((P-1) / r) & \cdots & \operatorname{sinc}(0 / r)
\end{array}\right]\left[\begin{array}{c}
c_{1} \\
\vdots \\
c_{P}
\end{array}\right]} \\
=\left[\begin{array}{c}
\operatorname{sinc}(1 / r) \\
\vdots \\
\operatorname{sinc}(P / r)
\end{array}\right]
\end{array}
$$

with $\operatorname{sinc}(x)=\sin (\pi x) /(\pi x)$. It should be noted that the autocorrelation matrix $\mathbf{R}$ in (43) becomes ill-conditioned as the compensation order increases. Some methods to closely approximate the solution to this system are described in [17].

The ill-conditioning of the matrix equation also leads to solutions with compensation coefficients that are large in magnitude, which can cause the resulting systems to be unstable for certain erasure conditions, as described in Section III-C. As discussed, stability in this case can be enforced by solving the constrained quadratic program of (37). Due to the shift invariance of the frame there is only one cost function to be minimized.

Fig. 4 shows simulation results that demonstrate the performance of the algorithms in the case of i.i.d. erasures. The input $a_{k}$ to the system is a white Gaussian process with unit variance and zero mean. The oversampling frame is approximated using a 4096th order, Hamming window FIR filter with cutoff $\pi / r$. Since the synthesis filter is not ideal, the feedback coefficients are calculated using the implemented filter autocorrelation instead of the autocorrelation of the ideal filter. To compute the error, the output is compared to the unerased signal, as synthesized using the low-pass filter. The subplots demonstrate the error power normalized by the signal power as a function of the probability of erasure. The region of instability is indicated in the figures by the trend of the corresponding plots to grow rapidly above the axis limit of $0 \mathrm{db}$, towards infinity, as the erasure rate increases.

In Fig. 4(a), the error due to erasures is plotted for various compensation orders, $P$, with constant redundancy $r=4$. Compensation order $P=0$ corresponds to a baseline uncompensated system. The compensation coefficients are computed to be optimal using (39). As the compensation order increases, the compensation error is improved at low probabilities of erasures 


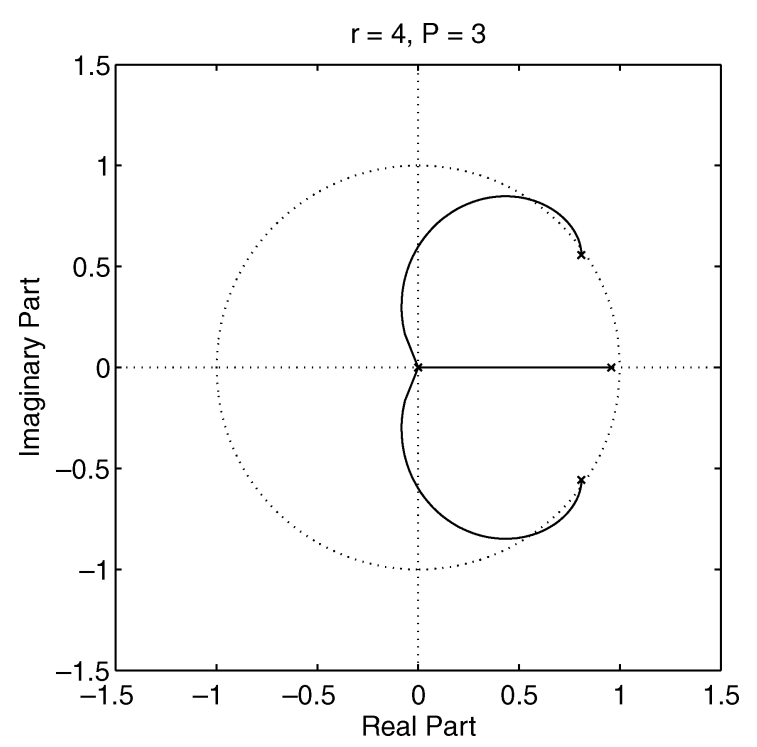

Fig. 5. Root locus plot of the system $H_{t}(z)=1 /\left(1-q \sum_{k=1}^{P} c_{k} z^{-k}\right)$ for $0 \leq$ $q \leq 1$, with the compensation coefficients computed using an ideal low-pass filter as a frame. At $q=0$ all poles are at the origin. As $q$ increases, two of the three poles (following the top and bottom trajectories) move out of the unit circle, making the system unstable. As $q$ reaches 1, the poles return inside and the system returns to stability.

but the system becomes unstable with a lower erasure probability. In Fig. 4(b), the error due to erasures is plotted for a fixed compensation order $P=2$ and various redundancy rates $r$. As the redundancy increases the compensation is improved, but, as expected, the systems are stable in a smaller range of erasure probabilities.

Fig. 4(c) and (d) demonstrates the tradeoff between compensation and stability. In both figures, the compensation order is $P=5$. The compensation coefficients are computed using the constraint $\sum_{k=1}^{P}\left|c_{k}\right|<\eta$. In Fig. 4(c), the redundancy is $r=$ 16 and $\eta$ varies, while in Fig. 4(d) $\eta=1.5$ and the redundancy is variable. The figures demonstrate that as the constraint is relaxed the compensation improves and the stability decreases. They further demonstrate that under a fixed constraint, as the redundancy increases, the compensation improves, while the regions of stability are essentially not affected.

The root locus plot for the system with transfer function $H_{t}(z)=1 /\left(1-q \sum_{k=1}^{P} c_{k} z^{-k}\right)$ is plotted in Fig. 5, which demonstrates the trajectory of the system poles as $q$, the probability of erasures, varies. The coefficients in the figure are computed for a $P=3$ order system with redundancy $r=4$. The plot demonstrates the stability in the mean of the system as the probability of erasures increases. This is a necessary condition for the stability of the algorithm and, therefore, when the poles of the system are outside the unit circle the algorithm is unstable. Furthermore, the stability of the system for $q=1$ demonstrates that the transmitter of Fig. 2(a) is stable, and, therefore, the separation of the algorithm to a transmitter/receiver combination is theoretically possible even if the operating conditions are such that the overall system is unstable. We should note that in practice the location of the poles so close to the unit circle for $q=1$ might cause

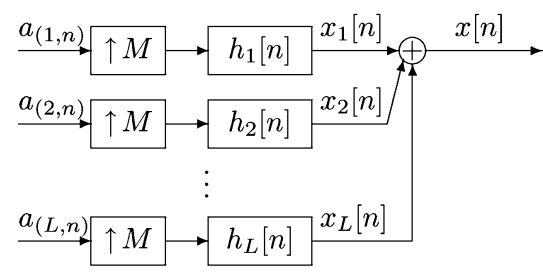

Fig. 6. A general synthesis filterbank.

dynamic range and finite-wordlength implementation issues, and, therefore, some compromise might still be necessary.

\section{B. Synthesis Using a Synthesis Filterbank}

This section examines the same compensation method applied to the synthesis frame implied by the $L$-channel oversampled filterbank of Fig. 6. In this example, the coefficients are transmitted in sequence, time-multiplexed in one-channel such that coefficient $a_{(l, n)}$ is transmitted at time $k=l L+n$, in which $l$ is the channel index, $n$ is the coefficient index within that channel and $k$ is the coefficient index in the single-channel stream. Erasures occur in individual coefficients and the compensation is causal in $k$. We use the notation $\mathbf{f}_{k}$ to denote the frame vectors indexed using the single stream sequence and the notation $\mathbf{f}_{(l, n)}=\mathbf{f}_{l L+n}$ to denote the vectors indexed using the two-dimensional channel-and-time indexing. Similar notation is adopted for all the relevant coefficients.

In this case, there are $L$ different sets of compensation coefficients that need to be solved for. Due to the shift invariance of the filters in each channel, the system $H$ in Fig. 1 and 2 becomes periodically time varying. Assuming $P^{t h}$ order compensation for each set of coefficients, the $L$ sets should each be chosen to minimize $\left\|\mathbf{f}_{l}-\sum_{m=1}^{P} c_{l, l+m} \mathbf{f}_{l+m}\right\|$, $l=0, \ldots, L-1$, either using a projection or subject to the stability constraints discussed in Section III-C. It should be noted that the stability constraints are on sums of coefficients taken from all $L$ optimizations, and, therefore, the constrained optimization problems cannot be solved independently. In this example, the $L$ competing cost functions are jointly optimized by minimizing the sum of their squared norms $\sum_{l=0}^{L-1}\left\|\mathbf{f}_{l}-\sum_{m=1}^{P} c_{l, l+m} \mathbf{f}_{l+m}\right\|^{2}$ subject to the constraints. Although the issue of joint optimization of the cost functions deserves further investigation, such investigation is beyond the scope of this paper

If the filters of the filterbank have finite length $N$, and their impulse responses span the space of length- $N$ signals, then compensation beyond a certain order does not reduce the error further. Specifically, it can be verified that for frame vector $\mathbf{f}_{l}, l=0, \ldots, L-1$ only the next $P=N+L-l-1$ are necessary to minimize the error. If $M=N$, it can be further shown that only $P=L-l-1$ frame vectors subsequent to $\mathbf{f}_{l}$ are useful for compensation. For $M \leq N$, this generalizes to $P=\lceil N / M\rceil L-l-1$ subsequent vectors. To satisfy the span condition, the number of filters in the filterbank should be $L \geq N$.

The filterbank used to generate the results in this section has $L$ filters of length $N \leq L$. The nonzero part of the impulse 
(a) $M=1$

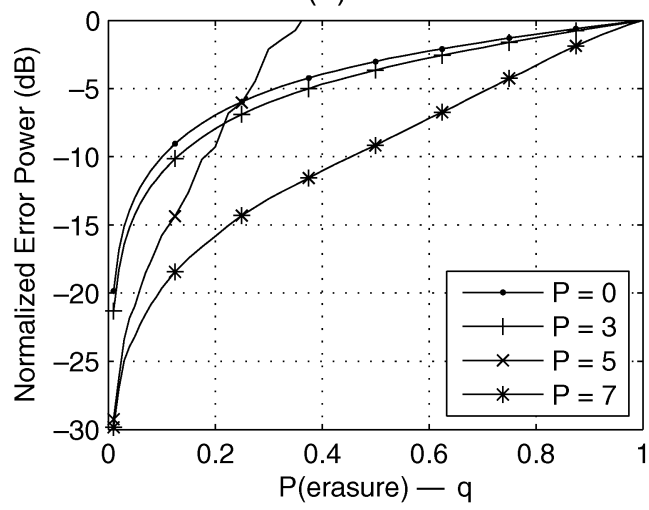

(c) $M=2$

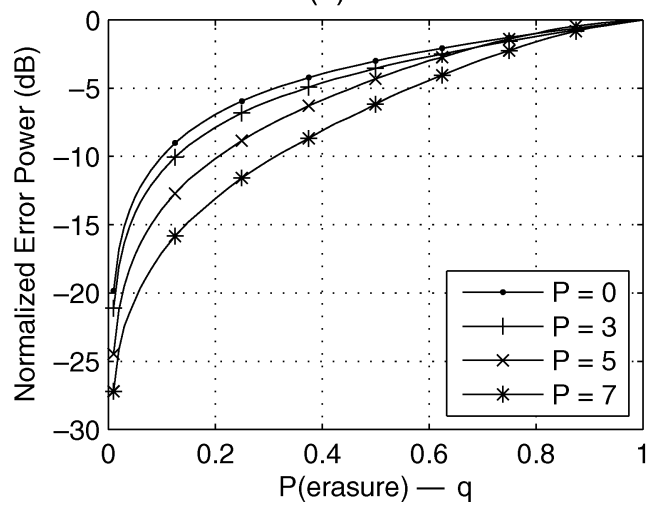

(b) $M=1, \eta=1$

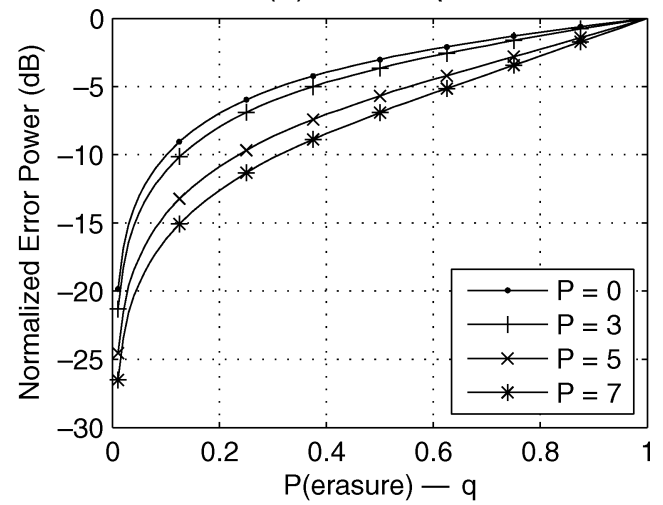

(d) $M=2, \eta=1$

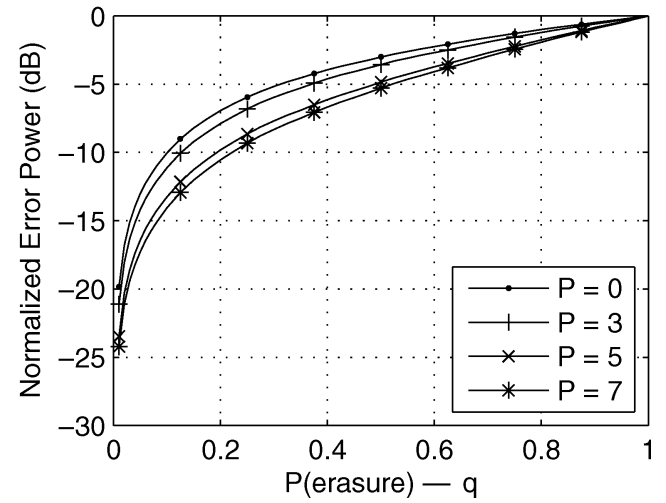

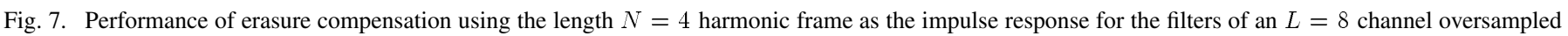

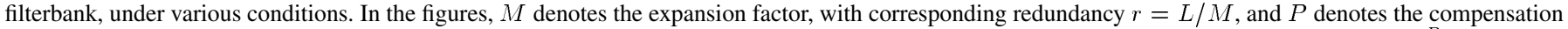

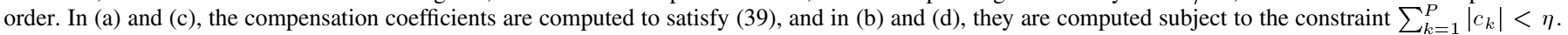
(a) $\mathrm{M}=1$; (b) $\mathrm{M}=1, \eta=1$; (c) $\mathrm{M}=2$; (d) $\mathrm{M}=2, \eta=1$.

response of the $l^{\text {th }}$ filter is equal to the $l^{\text {th }}$ vector of an $L$-vector Harmonic frame for $\mathbb{R}^{K}$ [25]. Specifically, for $N$ even

$$
\begin{aligned}
h_{l}[2 n] & =\sqrt{\frac{2}{N}} \cos \frac{2 \pi(n+1) l}{L}, n=0, \ldots, \frac{N}{2}-1, \\
h_{l}[2 n+1] & =\sqrt{\frac{2}{N}} \sin \frac{2 \pi(n+1) l}{L}, n=0, \ldots, \frac{N}{2}-1 \\
h_{l}[n] & =0, \text { otherwise. }
\end{aligned}
$$

For $N$ odd

$$
\begin{aligned}
h_{l}[n] & =\frac{1}{\sqrt{N}}, n=0 \\
h_{l}[2 n-1] & =\sqrt{\frac{2}{N}} \cos \frac{2 \pi n l}{L}, n=1, \ldots, \frac{N}{2} \\
h_{l}[2 n] & =\sqrt{\frac{2}{N}} \sin \frac{2 \pi n l}{L}, n=1, \ldots, \frac{N}{2} \\
h_{l}[n] & =0, \text { otherwise. }
\end{aligned}
$$

The harmonic frame has the property that any subset of $N$ frame vectors spans $\mathbb{R}^{N}[25]$. This property guarantees that erasures of the coefficients corresponding to the top $L-N$ channels can be perfectly compensated for using the causal system described. Furthermore, the impulse responses span the space of length- $N$ signals and the compensation has finite length. Specifically, compensation order beyond $P=2 N-1$ should not provide any compensation benefit. The input to the systems is assumed to be a white, unit variance Gaussian process. The redundancy of this filterbank is $r=L / M$.

Fig. 7 demonstrates the simulation results for an $L=8$ channel system with synthesis filters of length $N=4$ for various compensation orders $P$. The synthesis bank for Fig. 7(a) and (b) has an expansion factor of $M=1$, i.e., the system has redundancy $r=L / M=8$. For the bottom parts, Fig. 7(c) and (d), the expansion factor is $M=2$, with corresponding redundancy $r=L / M=4$. The simulations are performed assuming optimal compensation using (6) in Fig. 7(a) and (c). In Fig. 7(b) and (d), the compensation is performed using the stability constraint with $\eta=1$. The compensation orders $P$ were selected to illustrate a number of issues. In all simulations increasing the order beyond $P=7$ provides no compensation benefit, as expected.

In Fig. 7(a), it is demonstrated that although the system is unstable for compensation order $P=5$, the system returns to stability as the order increases to $P=7$, even though the stability constraint is not met. Imposing the stability constraint in Fig. 7(b) makes the $P=5$ order system stable but reduces the gain from compensation. Although not shown in the figure, $P=4$ and $P=6$ order systems also exhibit unstable behavior when the constraint is not imposed. 
The same issue is demonstrated in Fig. 7(c) and (d). The optimal compensation, although stable, does not meet the constraint for all the compensation orders shown. The system is unstable for compensation order $P=6$, not shown in the figure. Imposing the constraint ensures stability, but unnecessarily penalizes the compensation ability of the system. In these figures, it is also evident that decreasing the system redundancy by making $M=2$ reduces the compensation ability of the system.

\section{APPENDIX A}

\section{INPUT-OUTPUT EQUIVALENCE OF THE SYSTEMS IN FIG. 1 AND 2}

To show that the system in Fig. 2 implements the same compensation method as the system in Fig. 1, it suffices to examine the evolution of the coefficients

$$
\begin{aligned}
a_{k}= & a_{k}^{\prime}-\sum_{m=1}^{P} c_{k-m, k} a_{k-m}^{\prime} \\
= & a_{k}^{\prime}-\sum_{m=1}^{P} c_{k-m, k} a_{k-m}^{\prime} e_{k-m} \\
& -\sum_{m=1}^{P} c_{k-m, k} a_{k-m}^{\prime}\left(1-e_{k-m}\right) \\
\check{a}_{k}= & \sum_{m=1}^{P} c_{k-m, k} a_{k-m}^{\prime \prime} \\
= & \sum_{m=1}^{P} c_{k-m, k} a_{k-m}^{\prime} e_{k-m} \\
& +\sum_{m=1}^{P} c_{k-m, k} \check{a}_{k-m}\left(1-e_{k-m}\right) .
\end{aligned}
$$

Rearranging (52) and substituting into (54)

$$
\begin{aligned}
\check{a}_{k}= & a_{k}^{\prime}-\sum_{m=1}^{P} c_{k-m, k} a_{k-m}^{\prime}\left(1-e_{k-m}\right)-a_{k} \\
& +\sum_{m=1}^{P} c_{k-m, k} \check{a}_{k-m}\left(1-e_{k-m}\right) \\
\Rightarrow a_{k}= & a_{k}^{\prime}-\check{a}_{k} \\
& -\sum_{m=1}^{P} c_{k-m, k}\left(a_{k-m}^{\prime}-\check{a}_{k-m}\right)\left(1-e_{k-m}\right) \\
\Leftrightarrow a_{k}^{\prime}-\check{a}_{k}= & \sum_{m=1}^{P} c_{k-m, k}\left(a_{k-m}^{\prime}-\check{a}_{k-m}\right)\left(1-e_{k-m}\right)+a_{k}
\end{aligned}
$$

which holds for any input $a_{k}$ and any signal $e_{k}$, not restricted to be an erasure pattern of zeros and ones. Assuming the same initial conditions, and comparing with (21), it follows that:

$$
\tilde{a}_{k}=a_{k}^{\prime}-\check{a}_{k}, \text { for all } k \text {. }
$$

Using (27) in (58), the output $\hat{a}_{k}$ is equal to

$$
\hat{a}_{k}=a_{k}^{\prime} e_{k}-\check{a}_{k} e_{k}=\tilde{a}_{k} e_{k}
$$

which is the same as (22). Thus, the two systems are inputoutput equivalent.

\section{APPENDIX B \\ Proof OF THE StABILITY RESUlts}

Taking the square root of the second moment in (21) and applying the triangle inequality on the random variables it follows that:

$$
\begin{array}{r}
\sqrt{E\left\{\tilde{a}_{k}^{2}\right\}} \leq \sum_{m=1}^{P}\left|c_{k-m, k}\right| \cdot \sqrt{E\left\{\left(1-e_{k-m}\right)^{2} \tilde{a}_{k-m}^{2}\right\}} \\
+\sqrt{E\left\{a_{k}^{2}\right\}} .
\end{array}
$$

Assuming that

$$
E\left\{\left(1-e_{k-m}\right)^{2} \tilde{a}_{k-m}^{2}\right\}=E\left\{\left(1-e_{k-m}\right)^{2}\right\} E\left\{\tilde{a}_{k-m}^{2}\right\}
$$

and that the probability of erasure is $q$, (62) follows:

$$
\sqrt{E\left\{\tilde{a}_{k}^{2}\right\}} \leq \sum_{m=1}^{P} \sqrt{q}\left|c_{k-m, k}\right| \cdot \sqrt{E\left\{\tilde{a}_{k-m}^{2}\right\}}+\sqrt{E\left\{a_{k}^{2}\right\}} .
$$

It should be noted that $E\left\{\left(1-e_{k-m}\right)^{2} \tilde{a}_{k-m}^{2}\right\}=q E\left\{\tilde{a}_{k-m}^{2}\right\}$ for any joint density of the $e_{k}$, as long as the unconditional mean of $1-e_{k}$ is constant and equal to $q$, and $e_{k}$ is independent of $\tilde{a}_{k}$, conditional on the previous values $e_{k-i}$ and $\tilde{a}_{k-i}$, for $i>0$. Independence of the erasure process $e_{k}$ with the frame expansion coefficients $a_{k}$ is sufficient to guarantee the conditional independence, for any joint density of the erasure sequence $e_{k}$ and for any correlation structure of the frame expansion coefficients $a_{k}$.

The systems in (29) and (30) follow similar dynamics within a factor of $\sqrt{q}$ in the coefficients. BIBO stability of these systems can be guaranteed if the sum of the coefficients is slightly less than 1 . In the case of (29), stability can be guaranteed using (31) if

$$
\sum_{m=1}^{P}\left|c_{k-m, k}\right| \leq 1-\epsilon
$$

Substituting (63) into (64), stability follows by induction, assuming initial conditions are within some bound $B$

$$
\begin{aligned}
\left|\tilde{a}_{k}\right| & \leq \sum_{m=1}^{P}\left|c_{k-m, k}\right| \cdot\left|\tilde{a}_{k-m}\right|+\left|a_{k}\right| \\
& \leq \sum_{m=1}^{P}\left|c_{k-m, k}\right| B / \epsilon+B \\
\Rightarrow\left|\tilde{a}_{k}\right| & \leq B / \epsilon .
\end{aligned}
$$

Similarly the stability in (30) is guaranteed if the sum of the coefficient magnitudes is less than $\eta=1 / \sqrt{q}$

$$
\sum_{m=1}^{P}\left|c_{k-m, k}\right|<\eta=1 / \sqrt{q} .
$$




\section{REFERENCES}

[1] I. Daubecies, Ten Lectures on Wavelets, ser. CBMS-NSF Regional Conference Series in Applied Mathematics. Philadelphia, PA: SIAM, 1992.

[2] O. Christensen, An Introduction to Frames and Riesz Bases. Boston, MA: Birkhäuser, 2002.

[3] S. G. Mallat and Z. Zhang, "Matching pursuits with time-frequency dictionaries," IEEE Trans. Signal Process., vol. 41, no. 12, pp. 3397-3415, Dec. 1993.

[4] S. S. Chen, D. L. Donoho, and M. A. Saunders, "Atomic decomposition by basis pursuit," SIAM J. Sci. Comp., vol. 20, no. 1, pp. 33-61, 1999.

[5] V. K. Goyal, J. Kovačević, and J. A. Kelner, "Quantized frame expansions with erasures," Appl. Comput. Harm. Anal., vol. 10, pp. 203-233, 2001.

[6] J. Kovačević, P. L. Dragotti, and V. K. Goyal, "Filter bank frame expansions with erasures," IEEE Trans. Inf. Theory, vol. 48, no. 6, pp. 1439-1450, Jun. 2002.

[7] R. Bernardini and R. Rinaldo, "Efficient reconstruction from framebased multiple descriptions," IEEE Trans. Signal Process., vol. 53, no. 8, pp. 3282-3296, Aug. 2005.

[8] P. G. Casazza and J. Kovačević, "Equal-norm tight frames with erasures," Adv. Computat. Math., Special Issue on Frames, pp. 387-430, 2003.

[9] Z. Cvetkovic, "Resilience properties of redundant expansions under additive noise and quantization," IEEE Trans. Inf. Theory, vol. 49, no. 3, pp. 644-656, Mar. 2003.

[10] T. Strohmer and R. Heath, "Grassmanian frames with applications to coding and communication," Appl. Comput. Harm. Anal., vol. 14, pp. 257-275, 2003.

[11] R. B. Holmes and V. I. Paulsen, "Optimal frames for erasures," Linear Algebra Its Applicat., no. 377, pp. 31-51, 2004.

[12] G. Rath and C. Guillemot, "Frame-theoretic analysis of DFT codes with erasures," IEEE Trans. Signal Process., vol. 52, no. 2, pp. 447-460, Feb. 2004.

[13] B. G. Bodmann and V. I. Paulsen, "Frames, graphs and erasures," Linear Algebra Its Applicat., no. 404, pp. 118-146, 2005.

[14] M. Püschel and J. Kovačević, "Real, tight frames with maximal robustness to erasures," in IEEE Proc. Data Compression Conf. (DCC) 2005, Snowbird, UT, 2005, pp. 63-72.

[15] G. Romano, P. Salvo, and F. Palmieri, "Optimal correlating transform for erasure channels," IEEE Signal Process. Lett., vol. 12, no. 10, pp. 677-680, Oct. 2005.

[16] P. Boufounos and A. V. Oppenheim, "Quantization noise shaping on arbitrary frame expansions," EURASIP J. Appl. Signal Process., Special Issue on Frames and Overcomplete Represent. Signal Process., Commun., Inf. Theory, vol. 2006, 2006, Article ID 53807, DOI:10. 1155/ASP/2006/53807, $12 \mathrm{pp}$.

[17] S. R. Dey, A. I. Russell, and A. V. Oppenheim, "Pre-compensation for anticipated erasures in LTI interpolation systems," IEEE Trans. Signal Process., vol. 54, no. 1, pp. 325-335, Jan. 2005.

[18] I. F. Akyildiz, S. Weilian, Y. Sankarasubramaniam, and E. Cayirci, "A survey on sensor networks," IEEE Commun. Mag., vol. 40, no. 8, pp. 102-114, Aug. 2002.

[19] M. Said and A. V. Oppenheim, "Discrete time randomized sampling," in Proc. IEEE ICECS 2001, Malta, Sep. 2001, vol. 3, pp. 1407-1411.

[20] S. R. Dey and A. V. Oppenheim, "Frequency shaped randomized sampling," in Proc. IEEE ICASSP 2007, Honolulu, HI, Apr. 2007, to be published.

[21] G. U. Yule, "On a method of investigating periodicities in disturbed series, with special reference to Wolfer's sunspot numbers," Philosoph. Trans. Roy. Soc. London, Series A, vol. 226, pp. 267-298, 1927.

[22] G. Walker, "On periodicity in series of related terms," Proc. Roy. Soc. London, Series A, vol. 131, no. 818, pp. 518-532, Jun. 1931.

[23] N. Levinson, "The wiener RMS (root mean square) error criterion in filter design and prediction," J. Math. Phys., vol. 25, no. 4, pp. 261-278, Jan. 1947.

[24] J. Durbin, "The fitting of time-series models," Revue de l'Institut International de Statistique, vol. 28, no. 3, pp. 233-244, 1960.

[25] V. K. Goyal, M. Vetterli, and N. T. Thao, "Quantized overcomplete expansions in $\mathbb{R}^{N}$ : Analysis, synthesis, and algorithms," IEEE Trans. Inf. Theory, vol. 44, no. 1, pp. 16-31, Jan. 1998.

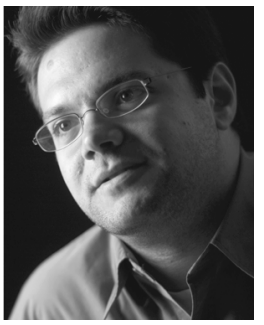

Petros Boufounos (S'02-M'06) received the S.B. degree in economics in 2000, the S.B. and M.Eng. degrees in electrical engineering and computer science (EECS) in 2002, and the Sc.D. degree in EECS in 2006, all from the Massachusetts Institute of Technology (MIT), Cambridge.

Since September 2006, he has been with the Digital Signal Processing Group, Rice University, Houston, TX, conducting research in the area of compressive sensing. In addition to compressive sensing, his research interests include signal processing, data representations, frame theory, and machine learning applied to signal processing.

Dr. Boufounos has received the Ernst A. Guillemin Master Thesis Award for his work on DNA sequencing and the Harold E. Hazen Award for Teaching Excellence, both from the MIT EECS Department. He has also been an MIT Presidential Fellow. He is a member of Sigma Xi, Eta Kappa Nu, and Phi Beta Kappa.

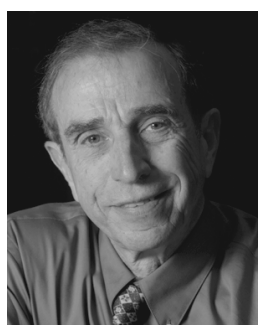

Alan V. Oppenheim (S'67-M'65-SM'71-F'77LF'03) was born in New York, NY, on November 11, 1937. He received the S.B. and S.M. degrees in 1961 and the Sc.D. degree in 1964, all in electrical engineering, from the Massachusetts Institute of Technology (MIT), Cambridge. He is also the recipient of an honorary doctorate from Tel Aviv University.

In 1964, he joined the faculty at MIT, where he is currently the Ford Professor of Engineering and a MacVicar Faculty Fellow. Since 1967, he has been affiliated with MIT Lincoln Laboratory and since 1977, with the Woods Hole Oceanographic Institution, MA. His research interests are in the general area of signal processing and its applications. He is coauthor of the widely used textbooks Discrete-Time Signal Processing (Upper Saddle River, NJ: Prentice-Hall, 1999, 2nd ed.) and Signals and Systems (Upper Saddle River, NJ: Prentice-Hall, 1996,2 nd ed.). He is also editor of several advanced books on signal processing.

Dr. Oppenheim is a member of the National Academy of Engineering and a member of Sigma Xi and Eta Kappa Nu. He has been a Guggenheim Fellow and a Sackler Fellow. He has also received a number of awards for outstanding research and teaching, including the IEEE Education Medal, the IEEE Signal Processing Society Signal Processing Education Award, the IEEE Centennial Award, the IEEE Third Millennium Medal, the Society Award, the Technical Achievement Award and the Senior Award of the IEEE Society on Acoustics, Speech and Signal Processing. He is the recipient of the 2007 IEEE Jack S. Kilby Signal Processing Medal. He has also received a number of awards at MIT for excellence in teaching, including the Bose Award and the Everett Moore Baker Award.

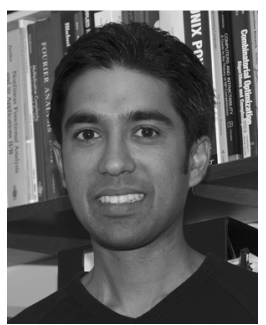

Vivek K Goyal (S'92-M'98-SM'03) received the B.S. degree in mathematics and the B.S.E. degree in electrical engineering (both with highest distinction) from the University of Iowa, Iowa City, in 1993. He received the M.S. and Ph.D. degrees in electrical engineering from the University of California, Berkeley, in 1995 and 1998, respectively.

He was a Research Assistant with the Laboratoire de Communications Audiovisuelles at École Polytechnique Fédérale de Lausanne, Switzerland, in 1996, a Member of Technical Staff in the Mathematics of Communications Research Department of Bell Laboratories, Lucent Technologies, during 1998-2001, and a Senior Research Engineer for Digital Fountain, Inc., from 2001 to 2003. He is currently the Esther and Harold E. Edgerton Assistant Professor of Electrical Engineering and a member of the Research Laboratory of Electronics at the Massachusetts Institute of Technology, Cambridge. His research interests include source coding theory, sampling, quantization, and information gathering and dispersal in networks.

Dr. Goyal is a member of Phi Beta Kappa, Tau Beta Pi, Sigma Xi, Eta Kappa $\mathrm{Nu}$, SIAM, and ACM. In 1998, he received the Eliahu Jury Award of the University of California, Berkeley, awarded to a graduate student or recent alumnus for outstanding achievement in systems, communications, control, or signal processing. He was also awarded the 2002 IEEE Signal Processing Society Magazine Award and an NSF CAREER Award. He serves on the IEEE Signal Processing Society's Image and Multiple Dimensional Signal Processing Technical Committee and as permanent Co-Chair of the SPIE Wavelets conference series. 